\title{
BMJ Open Does preoperative rehabilitation for patients planning to undergo joint replacement surgery improve outcomes? A systematic review and meta-analysis of randomised controlled trials
}

\author{
Li Wang, ${ }^{1,2,3}$ Myeongjong Lee, ${ }^{4}$ Zhe Zhang, ${ }^{5}$ Jessica Moodie, ${ }^{1}$ Davy Cheng, ${ }^{1,6}$ \\ Janet Martin ${ }^{1,6,7}$
}

To cite: Wang L, Lee $M$, Zhang Z, et al. Does preoperative rehabilitation for patients planning to undergo joint replacement surgery improve outcomes? A systematic review and metaanalysis of randomised controlled trials. BMJ Open 2016;6:e09857.

doi:10.1136/bmjopen-2015009857

- Prepublication history and additional material is available. To view please visit the journal (http://dx.doi.org/ 10.1136/bmjopen-2015009857).

Received 28 August 2015 Revised 24 November 2015 Accepted 10 December 2015

CrossMark

For numbered affiliations see end of article.

Correspondence to

Dr Janet Martin;

jmarti83@uwo.ca

\section{ABSTRACT}

Objectives: The clinical impact of preoperative physiotherapy on recovery after joint replacement remains controversial. This systematic review aimed to assess the clinical impact of prehabilitation before joint replacement.

Design: We searched PubMed, Embase and Cochrane CENTRAL up to November 2015 for randomised controlled trials comparing prehabilitation versus no prehabilitation before joint replacement surgery. Postoperative pain and function scores were converted to Western Ontario and McMaster Universities Osteoarthritis Index (WOMAC) pain and function subscales $(0-100$, high scores indicate worse outcome). Random effects meta-analysis was performed to calculate weighted mean differences (WMD, 95\% Cl), subgrouped by hip and knee surgery. Primary and secondary outcomes: Postoperative pain and function scores, time to resume activities of daily living, quality of life, length of hospital stay, total cost, patient satisfaction, postoperative complications, any adverse events and discontinuations.

Results: Of 22 studies (1492 patients), 18 had high risk of bias. Prehabilitation slightly reduced pain scores within 4 weeks postoperatively (WMD -6.1 points, $95 \% \mathrm{Cl}-10.6$ to -1.6 points, on a scale of $0-100$ ), but differences did not remain beyond 4 weeks.

Prehabilitation slightly improved WOMAC function score at $6-8$ and 12 weeks (WMD $-4.0,95 \% \mathrm{Cl}-7.5$ to -0.5 ), and time to climbing stairs (WMD -1.4 days, $95 \% \mathrm{Cl}-1.9$ to -0.8 days), toilet use ( -0.9 days, $95 \%$ $\mathrm{Cl}-1.3$ to -0.5 days) and chair use (WMD -1.2 days, $95 \% \mathrm{Cl}-1.7$ to -0.8 days). Effects were similar for knee and hip surgery. Differences were not found for SF-36 scores, length of stay and total cost. Other outcomes of interest were inadequately reported.

Conclusions: Existing evidence suggests that prehabilitation may slightly improve early postoperative pain and function among patients undergoing joint replacement; however, effects remain too small and short-term to be considered clinically-important, and did not affect key outcomes of interest (ie, length of stay, quality of life, costs).

\section{Strengths and limitations of this study}

- The methodology was rigorous, and included a comprehensive systematic search-without limits by language, date or publication status-that identified seven randomised controlled trials (RCTs) not included in any previous systematic reviews.

- We went beyond previous published systematic reviews by analysing the effect of prehabilitation by converting to a standardised measurement of Western Ontario and McMaster Universities Osteoarthritis Index (WOMAC) pain and function scores, and used different presentation methods to enhance interpretability and to improve ability to find potential signals in effect size through meta-analysis.

- This meta-analysis addressed all available clinically relevant outcomes, while previous reviews addressed only a few selected outcomes. Application of GRADE for rating quality of evidence provides improved context for interpreting the findings in light of inherent strengths and limitations of the included studies.

- Compliance with prehabilitation was problematic in some studies, and was not reported in a number of studies.

\section{INTRODUCTION}

Total joint replacement surgery is considered as one of the most successful medical interventions, with significant pain relief and improvement in physical function and quality of life for patients with severe osteoarthritis. ${ }^{1}$ However, the recovery for a significant proportion of patients remains difficult and prolonged, and many never gain optimal functionality postoperatively. ${ }^{2-4}$ Therefore, researchers, clinicians and policymakers are still looking for better ways to improve the timelines and extent of recovery for patients undergoing total joint replacement. 
Physiotherapy has been delivered to patients for rehabilitation, traditionally after total joint replacement. However, preoperative physiotherapy and exercise programmes (also known as 'prehabilitation') have been proposed as a potential way to expedite recovery times and improve overall extent of recovery in patients planning to undergo joint replacement. One recently published review recommended preoperative exercise to maintain or improve function and pain $;{ }^{5}$ however, this recommendation was based on only one narrative systematic review with indeterminate effects. ${ }^{6}$ Although it seems intuitive that prehabilitation should improve patient disposition at the time of surgery, and may prepare patients for a better recovery after surgery, significant uncertainties remain about the overall balance of benefits and risks (and costs) for prehabilitation.

A number of related systematic reviews and meta-analyses have been published in the recent decade, with inconsistent methods and varied conclusions. ${ }^{6-13}$ Two papers suggested that prehabilitation reduced pain for patients undergoing joint replacement, ${ }^{8}{ }^{11}$ and improved physical function for patients undergoing hip replacement surgery, but not knee replacement surgery, ${ }^{8}$ while the remainder suggested prehabilitation did not clearly demonstrate beneficial effects or were unable to provide definitive conclusions. ${ }^{6} 79101213$ Furthermore, significant methodological limitations or errors have been identified among the existing systematic reviews. Some only qualitatively summarised the results; ${ }^{6} 79$ 11-13 two other meta-analyses ${ }^{810}$ are outdated, or mistakenly included some trials in which postoperative outcomes were not reported. Thus, we conducted an updated methodologically rigorous systematic review with meta-analysis to clarify whether evidence supports prehabilitation for patients planning to undergo joint replacement.

\section{METHODS}

\section{Eligibility criteria}

We systematically searched three databases up to 10 November 2015, including PubMed, Embase and Cochrane Central Register of Controlled Trials (CENTRAL). Eligible studies had to be randomised controlled trials comparing preoperative rehabilitation programmes (ie, prescribed and supervised exercises or physiotherapy with or without cointerventions such as education, nutritional counselling, acupuncture, transcutaneous electrical nerve stimulation, etc) versus no formal preoperative rehabilitation programmes, reporting at least one clinically-relevant outcome of interest during the postoperative period. Clinical outcomes of interest included postoperative pain scores (Visual Analogue Scale (VAS), or pain subcomponents of Western Ontario and McMaster Universities osteoarthritis index (WOMAC) or pain-related subdomains of other instruments), patient functionality (WOMAC function score, SF-36 physical functioning subdomain or other function-related instruments), time to resume activities of daily living (ADL), quality of life, patient satisfaction, infection, transfusions, stroke and death or overall postoperative complications. Resource-related outcomes of interest included hospital length of stay, readmissions and total hospital costs or total health system costs. Timeframes of relevance included in-hospital outcomes, as well as clinical or resource-related outcomes over the longer term, postoperatively.

Search terms included MeSH and keyword terms for exercise, prehabilitation, physiotherapy, physical therapy, activity, weight training, weight lifting, aquatic, swimming, strength training, endurance training, cycling, biking, kinesiotherapy, hydrotherapy, fitness, orthopaedic surgery and joint replacement and 'random*'. Limitations were placed neither on date of publication nor on language. Detailed search strategies are provided in the Appendix.

\section{Literature screening and data extraction}

Two reviewers (ML, ZZ) independently screened the articles by title and abstract using the predetermined eligibility criteria. Any disagreements were resolved by a third reviewer (LW). The third reviewer (LW) also checked all the reference lists of existing systematic reviews or meta-analyses and other reviews for potentially additional eligible articles.

Two reviewers (ML, JM) independently assessed the risk of bias of the included trials using the methods recommended by Cochrane Collaboration, ${ }^{14}$ including random sequence generation, allocation concealment, missing or incomplete outcome data, and blinding of patients, study personnel and outcome assessors. Any discrepancies were resolved by the third reviewer (LW).

Standardised data extraction forms were developed to specify the study characteristics, patient characteristics and outcomes. Three reviewers (ML, ZZ and LW) extracted the data. Data were verified by a fourth reviewer (JM).

\section{Statistical analysis}

Meta-analysis was performed using the random effects model. For discrete outcomes, relative risk (RR) and 95\% CIs (RR, 95\% CI) were calculated. For continuous outcomes, for example, pain score and function score, weighted mean differences (WMD, 95\% CI) were calculated after conversion to the Western Ontario and McMaster Universities osteoarthritis index (WOMAC) pain score (0-100) and WOMAC function score (0$100)$, in which a higher score indicates worse outcome. Sensitivity analysis was conducted by calculating standardised mean differences (SMD) and ratio of means (RoM).

If different pain scores were reported in one article (eg, WOMAC pain, SF-36 pain score), the WOMAC pain score was preferentially used. If WOMAC pain score was not reported, the pain score reported in the study was converted to a WOMAC pain score to allow for 
comparison across studies, and to allow for estimation of overall effect size. ${ }^{15}$ If pain scores were reported at rest and during activity, the pain score during activity was preferentially used for analysis. If pain scores were reported during different types of activities, the largest change of pain score during the most active movement was used preferentially. If different function scores were reported, the WOMAC function score was used preferentially for analysis. In the absence of WOMAC function scores, the alternate function score provided in the study was converted to a WOMAC function score. ${ }^{15}$ Two studies $^{16} 17$ only reported total scores of Hospital for Special Surgery Knee Rating System (HSSK) and WOMAC, respectively. Given the function score accounting for most of the total score and with similar trends of change over time as total score, we used the total score to replace the function score. To test whether this changed the effect size, sensitivity analysis was performed after removing the total scores from function measures to recalculate effect size.

To improve clinical relevance and interpretation of the results for postoperative pain and function improvement, we also converted continuous data from WOMAC pain score and WOMAC function score to a RR for achieving a 'patient acceptable symptom state' (defined as the number of patients achieving the threshold pain score or function score at which patients consider themselves 'well' or 'satisfied') derived from previous research. ${ }^{18-21}$ To calculate the RR, we assumed a normal distribution of WOMAC pain or function scores for the intervention and control groups, and used a threshold of 30 on the WOMAC $0-100$ scale to represent the threshold for the patient acceptable symptom state. The proportion of patients in the intervention and control groups with WOMAC pain or function $\leq 30$ was then calculated and combined across studies to derive a pooled RR. ${ }^{15} 22$ Finally, to further add to clinical applicability of the patient reported outcomes, we calculated the risk difference for the number of patients achieving this threshold of $\leq 30$ per 100 patients, using the RR and median risk among the control groups in the included studies. $^{23}$ Subsequently, sensitivity analyses were performed to explore whether using different thresholds (20 and 40) changed the conclusions, since our threshold of 30 represents a compromise of 20-40, suggested in previous studies of hip or knee surgery over the short term or long term.

Heterogeneity was estimated using the $\chi^{2}$ test and $\mathrm{I}^{2}$ statistic. Predefined subgroup analyses included separate analysis for hip and knee surgery patients, to test the existing hypothesis from a previous systematic review that prehabilitation improves postoperative pain and function more among patients undergoing hip replacement than among patients with knee replacement. ${ }^{8}$ Publication bias was explored using both visual inspection of funnel plots and Egger's test only when there were at least 10 studies included in the meta-analysis. ${ }^{14}$
GRADE methodology was used to summarise certainty in estimates of effect (quality of evidence) in the critically important outcomes for decision-making, ${ }^{23-29}$ including WOMAC pain scores and function scores from early follow-up to 24 weeks after surgery.

\section{RESULTS}

\section{Studies identified}

Figure 1 outlines study inclusion and exclusion. A total of 399 titles and abstracts were screened for inclusion, of which 110 studies were collected in full-text for review. Of these, 88 were excluded for the following reasons: no prehabilitation arm (ie, education only or postoperative rehabilitation only, $n=46)$, not randomised $(n=21)$, duplicate studies $(n=4)$, no postoperative outcomes data $(n=9)$, no outcome of interest $(n=2)$, conference abstracts $(n=3)$

and protocol only $(n=3)$. In total, 22 randomised controlled trials (1492 patients) of prehabilitation versus no prehabilitation met the inclusion criteria. Twenty studies provided usable data for the meta-analysis, and 22 studies contributed qualitative or quantitative data.

\section{Description of included studies}

Among the 22 included studies, 8 studies were of patients undergoing total hip replacement; ${ }^{17}$ 30-36 12 studies included patients undergoing total knee replacement $^{1637-47}$ and two studies included either hip or knee replacement. ${ }^{48} 49$ Most studies were conducted in developed countries (North American and Europe), except for three in developing countries (Serbia, ${ }^{34}$ Thailand ${ }^{45}$ and Turkey $^{32}$ ). The median sample size of included studies was 54, ranging from 21 to 165 patients. Mean age ranged from 51 to 76 years (table 1 ).

Nine studies compared physiotherapist-supervised exercise plus home exercise versus no intervention, or

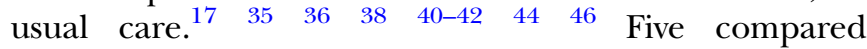
physiotherapist-supervised exercise versus no intervention, or usual care. ${ }^{16} 33373950$ Two studies compared home exercise only versus no intervention. ${ }^{30} 45$ Three studies compared physiotherapist-supervised exercise plus education versus no intervention. ${ }^{31} 3234$ One each compared physiotherapist supervised exercise plus education versus education, ${ }^{48}$ kinesiologist-supervised exercise versus placebo (kinesiologist supervised upper body exercise) ${ }^{43}$ and physiotherapist supervised exercise plus home exercise versus education plus home exercise, ${ }^{47}$ respectively, (see online supplementary table $\mathrm{S} 1$ ).

\section{Risk of bias}

Among the 22 trials, adequate sequence generation was reported in 17 trials $^{16} 30-3336-4345-48$ and allocation concealment was reported in 8 trials. ${ }^{30} 33373942434749$ The patients were blinded in one study, ${ }^{39}$ healthcare providers were blinded in three studies ${ }^{30} 3944$ and outcome assessors were blinded in 12 studies. $^{30-33} 36 \quad 374144 \quad 45 \quad 47-49$ Seventeen studies ${ }^{17} 30-34$ 36-40 $424346-49$ reported loss to follow-up, ranging from $1.7 \%$ to $65.3 \%$; among which the 
Figure 1 PRISMA flow diagram of study selection.

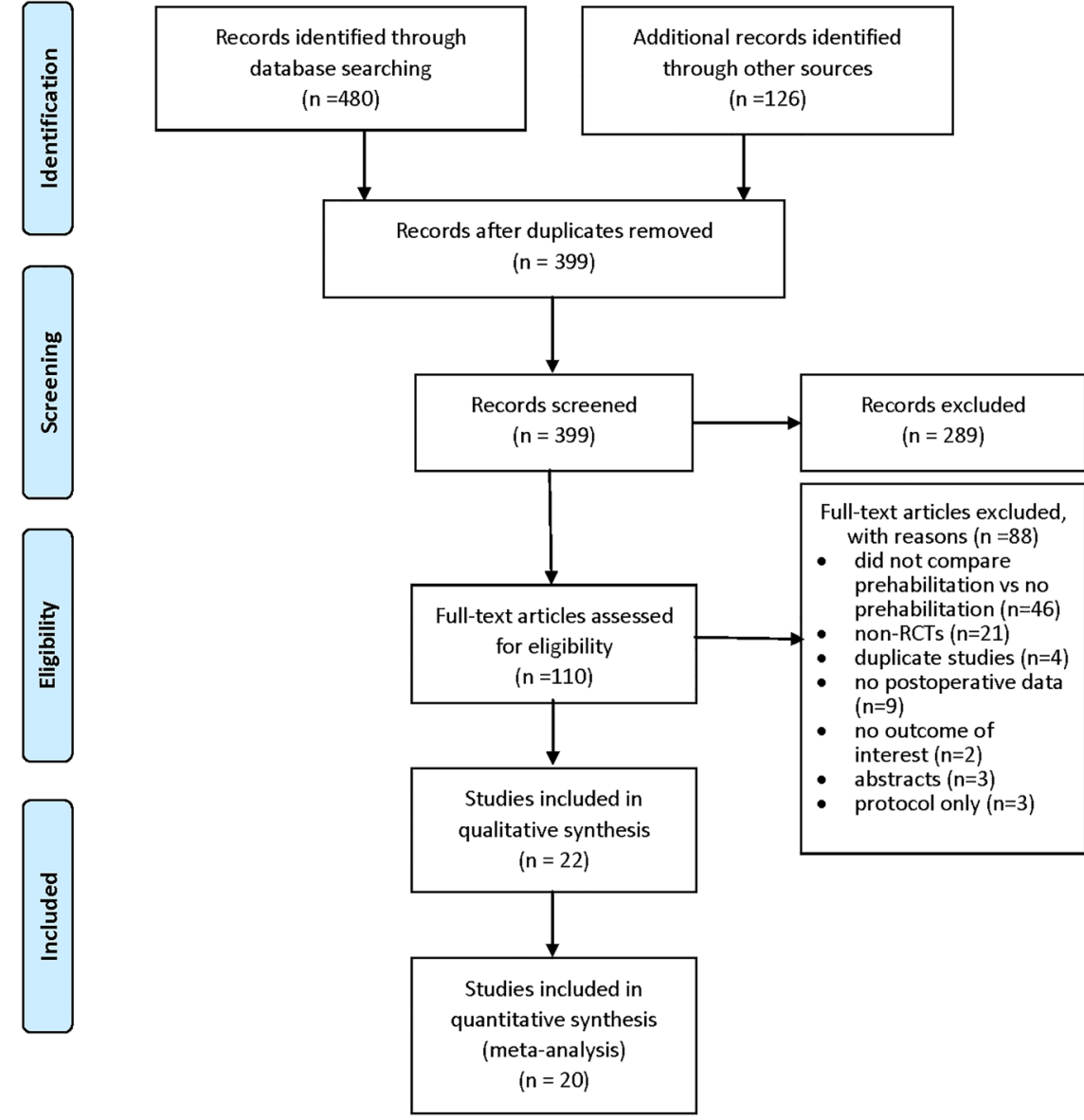

proportion of loss to follow-up was more than $15 \%$ in 10 studies. $^{30} \quad 34 \quad 36-39 \quad 42 \quad 43 \quad 47 \quad 48$ Ten of 17 studies with incomplete data used intention to treat analysis. ${ }^{30} 33363739424347-49$ Overall, 4 of the 22 included trials were rated as having a low risk of bias 333739 and 18 trials were rated as having a high risk of bias (table 2).

Online supplementary table S2 qualitatively summarises the major findings of included studies. In total, 22 studies described at least one clinical or resource-related benefit for prehabilitation versus control, and 18 studies $^{16} \quad 30-37 \quad 3940 \quad 42-48$ described no significant improvement for prehabilitation versus control. We conducted meta-analysis for pain scores, function scores, SF-36 PCS and MCS, hospital length of stay and total costs, based on data availability.

\section{Postoperative pain}

Fifteen trials with 18 comparisons and 1046 patients reported postoperative pain scores using different instruments, namely, WOMAC, ${ }^{31} 374042434548$ VAS, ${ }^{32} 34364447$ Knee injury and Osteoarthritis Outcome Score (KOOS) / Hip disability and Osteoarthritis Outcome Score (HOOS) ${ }^{49}$ and 10-graded scale. ${ }^{46}$ Only two trials ${ }^{31} 49$ reported significant improvement in pain at early follow-up ( $\leq 3$ months), including VAS pain at 3 months, ${ }^{31}$ and KOOS/HOOS pain at 6 weeks postoperatively, but not significant at 3 months. ${ }^{49}$
After converting to WOMAC pain $0-100$, prehabilitation significantly reduced postoperative pain at 4 weeks or less; however, the reduction of pain was clinically nominal (4 trials, 213 patients, WMD $-6.1,95 \%$ CI -10.6 to -1.6 , figure 2, GRADE: low certainty in estimates, tables 3 and 4). Differences in WOMAC pain scores after 4 weeks were no longer statistically significant for prehabilitation versus control (WOMAC pain score at $6-8$ weeks, 5 trials, 488 patients, WMD -1.4 , $95 \%$ CI -5.5 to +2.6 ; at 12 weeks, 10 trials, 806 patients, WMD $-2.9,95 \%$ CI -6.2 to +0.3 ; at 24 weeks, 3 trials, 247 patients, $-2.5,95 \%$ CI -5.6 to +0.6 ; at 1 year, 1 trial, 109 patients, WMD $-2.0,95 \%$ CI -7.5 to +3.5 ; GRADE: low to moderate certainty in estimates, tables 3 and 4 ).

When expressed as a RR, patients undergoing prehabilitation were more likely to achieve the acceptable pain state (WOMAC pain score $\leq 30$ ) with RR 1.09. When expressed as an absolute risk difference, $3.9 \%$ more patients with prehabilitation achieved the acceptable pain state (WOMAC pain score $\leq 30$ ) than patients without prehabilitation at 4 weeks (online supplementary table S3). However, this small difference would be considered clinically nominal. ${ }^{18-21}$

\section{Postoperative function}

Of 16 trials reporting on postoperative function, only four reported significant improvement in 
Table 1 Characteristics of included RCTs

\begin{tabular}{|c|c|c|c|c|c|c|c|}
\hline Study name & Patients, (n) & $\begin{array}{l}\text { Type of } \\
\text { surgery }\end{array}$ & Countries & Mean age & $\%$ Female & Mean BMI\% & Total OA \% \\
\hline Beaupre 2004 & 131 & TKR & Canada & 67 & 55 & 31.4 & NR \\
\hline Bitterli 2011 & 80 & THR & Switzerland & 66.9 & 38 & 27.4 & NR \\
\hline Brown 2012 & 32 & TKR & USA & NR & NR & 36.8 & NR \\
\hline D’Lima 1996 & 30 & TKR & USA & 69.8 & 46.6 & NR & 83.3 \\
\hline Evgeniadis 2008 & 48 & TKR & Greece & 68.3 & 76.3 & 34.1 & 100 \\
\hline Ferrara 2008 & 23 & THR & Italy & 63.4 & 60.8 & NR & 100 \\
\hline Gilbey 2003 & 76 & THR & Australia & 65.2 & 61.8 & 27.94 & NR \\
\hline Gocen 2004 & 60 & THR & Turkey & 51.3 & 35.5 & NR & 49 \\
\hline Gstoettner 2011 & 38 & TKR & Australia & 69.7 & 78.9 & 27.8 & 100 \\
\hline Hoogeboom 2010 & 21 & THR & The Netherlands & 76 & 66 & NR & NR \\
\hline Matassi 2014 & 122 & TKR & Italy & 66.5 & 48 & 28.5 & NR \\
\hline McKay 2012 & 22 & TKR & Canada & 61.3 & 59 & 34.3 & 100 \\
\hline Mitchell 2005 & 160 & TKR & UK & 70.3 & 57.9 & NR & 100 \\
\hline Oosting 2012 & 30 & THR & The Netherlands & 76 & 80 & 28.2 & 100 \\
\hline Rooks 2006 & 108 & THR/TKR & USA & 64.1 & 56 & 31.6 & 100 \\
\hline Topp 2009 & 54 & TKR & USA & 63.8 & 68 & 32.1 & 100 \\
\hline Tungtrongjit 2012 & 60 & TKR & Thailand & 64.5 & 83.3 & 24.8 & 100 \\
\hline Villadsen 2014 & 165 & THR/TKR & Denmark & 67 & 56 & 30.3 & 100 \\
\hline Vukomanovic 08 & 45 & THR & Serbia & 58.4 & 67 & NR & 100 \\
\hline Wang 2002 & 28 & THR & Australia & 67.1 & 64 & NR & 89 \\
\hline Weidenhielm 1993 & 39 & TKR & Sweden & 63.5 & 51.3 & 29.6 & 100 \\
\hline Williamson 2007 & 120 & TKR & UK & 69.8 & 52.9 & 32.7 & 100 \\
\hline
\end{tabular}

function, ${ }^{17} 314149$ including higher hip external rotation $^{31}$ or higher flexion range of motion scores, WOMAC physical function and total score, ${ }^{17}$ and less time to reach $90^{\circ}$ of knee flexion ${ }^{41}$ and greater improvement in $\mathrm{ADL}^{49}$ after surgery .

Sixteen trials (1118 patients) reported postoperative function scores, using different instruments, namely, WOMAC, ${ }^{17} 3137404243454748$ Harris hip score, ${ }^{32} 34$ SF-36 physical component summary (PCS), ${ }^{30}$ SF-36 physical functioning score, ${ }^{38}$ HSSK score, ${ }^{16}$ HOOS function in daily living ${ }^{36}$ and KOOS/HOOS ADL. ${ }^{49}$ After converting function scores to WOMAC function score (0-100), the difference was slightly improved (but numerically small on a scale of $0-100$ ) with prehabilitation versus no prehabilitation at early follow-up (WOMAC function score at $6-8$ weeks, 5 trials, 488 patients, WMD $-3.9,95 \%$ CI -7.6 to $-0.3, \mathrm{RR}=1.10$, figure 3 , GRADE: moderate certainty in estimates, tables 3 and 4), and at 12 weeks (12 trials, 836 patients, WMD $-4.0,95 \%$ CI -7.5 to -0.5 , $\mathrm{RR}=1.02$, figure 4, GRADE: very low certainty in estimates, tables 3 and 4). No significant difference for WOMAC function score was found after 12 weeks (at 24 weeks, 5 trials, 345 patients, WMD -0.5 , 95\% CI -5.8 to +4.7 ; at 1 year, 6 trials, 296 patients, WMD $-0.6,95 \%$ CI -2.6 to +1.5 , GRADE: low certainty in estimates, tables 3 and 4 ).

When expressed as a RR for achieving the acceptable threshold, the relative increases were small (RR 1.10 at 6-8 weeks; 1.02 at 12 weeks). When expressed as an absolute difference in likelihood of achieving the acceptable threshold, the differences ranged from $1.3 \%$ to $5.4 \%$ more patients achieving a WOMAC function score $\leq 30$ at $6-8$ weeks and 12 weeks, respectively, (online supplementary table S3).

\section{Resumption of ADL}

Resumption of ADL was rarely reported. In the two studies $(99 \text { patients })^{32} 34$ that reported ADL, meta-analysis suggested significantly earlier resumption of activities, including climbing stairs (2 trials, 99 patients, WMD -1.4 days, $95 \%$ CI -1.9 to -0.8 days), use of toilet ( 2 trials, 99 patients, -0.9 -day, $95 \%$ CI -1.3 to -0.5 days) and use of chair ( 2 trials, 99 patients, -1.2 days, $95 \%$ CI -1.7 to -0.8 days), but not for time to first day of walking (2 trials, 99 patients, -0.2-day, $95 \%$ CI -0.4 to +0.0-day), (table 3 ). However, based on the total time-course of recovery, the difference was small.

\section{Quality of life}

In nine studies, significant differences were not found for quality of life as reported in the SF-36, 3037394243 Quality of Well Being instrument, ${ }^{16}$ HOOS Hip-related quality of life, ${ }^{36}$ KOOS/HOOS Quality of Life subscale ${ }^{49}$ and Patient Specific Complaints (PSC) questionnaire. ${ }^{33} 36$ However, three trials reported a significant difference in quality of life scores within the first month, ${ }^{31} 3849$ including improved physical function scores or physical composite scores as reported in the SF-36 $6^{31}$ or EuroQol five Dimension Health Questionnaire (EQ5D). ${ }^{49}$ 


\begin{tabular}{|c|c|c|c|c|c|c|c|c|}
\hline & $\begin{array}{l}\text { Random sequence } \\
\text { generation }\end{array}$ & $\begin{array}{l}\text { Allocation } \\
\text { concealment }\end{array}$ & $\begin{array}{l}\text { Blinding of } \\
\text { patients }\end{array}$ & $\begin{array}{l}\text { Blinding of } \\
\text { healthcare } \\
\text { providers }\end{array}$ & $\begin{array}{l}\text { Blinding of } \\
\text { outcome } \\
\text { assessors }\end{array}$ & $\begin{array}{l}\text { Incomplete } \\
\text { outcome data }\end{array}$ & $\begin{array}{l}\text { Intention to treat } \\
\text { analysis }\end{array}$ & $\begin{array}{l}\text { Risk of } \\
\text { bias }\end{array}$ \\
\hline Beaupre 2004 & Yes & Yes & No & No & Yes & Yes, LTFU>15\% & Yes & Low risk \\
\hline Bitterli 2011 & Yes & Yes & No & Yes & Yes & Yes, LTFU>15\% & Yes & Low risk \\
\hline Brown 2012 & Yes & Unclear & No & Unclear & Unclear & Yes, LTFU>15\% & No & High risk \\
\hline D’Lima 1996 & Yes & Unclear & Unclear & Unclear & Unclear & No & Not applicable & High risk \\
\hline Evgeniadis 2008 & Yes & Yes & Yes & Yes & Unclear & Yes, LTFU>15\% & Yes & Low risk \\
\hline Ferrara 2008 & Yes & Unclear & No & No & Yes & Yes & No & High risk \\
\hline Gilbey 2003 & Unclear & Unclear & Unclear & Unclear & No & Yes & No & High risk \\
\hline Gocen 2004 & Yes & Unclear & No & Unclear & Yes & Yes & No & High risk \\
\hline Gstoettner 2011 & Yes & No & Unclear & Unclear & Unclear & Yes & No & High risk \\
\hline Hoogeboom 2010 & Yes & Yes & No & No & Yes & Yes & Yes & Low risk \\
\hline Matassi 2014 & Yes & Unclear & No & No & Yes & No & Not applicable & High risk \\
\hline McKay 2012 & Yes & Yes & Unclear & Unclear & Unclear & Yes, LTFU>15\% & Yes & High risk \\
\hline Mitchell 2005 & Yes & Yes & No & No & Unclear & Yes, LTFU>15\% & Yes & High risk \\
\hline Oosting 2012 & Yes & Unclear & No & No & Yes & Yes, LTFU>15\% & Yes & High risk \\
\hline Rooks 2006 & Yes & Unclear & Unclear & Unclear & Yes & Yes, LTFU>15\% & Yes & High risk \\
\hline Topp 2009 & Unclear & Unclear & No & Yes & Yes & No & Not applicable & High risk \\
\hline Tungtrongjit 2012 & Yes & Unclear & No & No & Yes & No & Not applicable & High risk \\
\hline Villadsen 2014 & Unclear & Yes & No & No & Yes & Yes & Yes & High risk \\
\hline Vukomanovic 08 & Unclear & Unclear & Unclear & Unclear & Unclear & Yes, LTFU>15\% & No & High risk \\
\hline Wang 2002 & Unclear & Unclear & Unclear & Unclear & No & No & Not Applicable & High risk \\
\hline Weidenhielm 1993 & Yes & Unclear & Unclear & Unclear & Unclear & Yes & No & High risk \\
\hline Williamson 2007 & Yes & Yes & No & No & Yes & Yes, LTFU>15\% & Yes & High risk \\
\hline
\end{tabular}

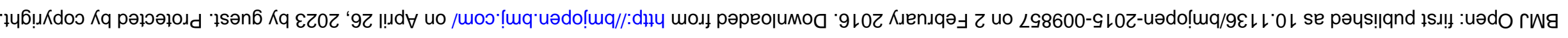






Figure 2 Pain score at 4 weeks or less (converted to WOMAC pain subscale $0-100$ ) for prehabilitation versus no prehabilitation in joint replacement surgery. WOMAC, Western Ontario and McMaster Universities Osteoarthritis Index.

Nevertheless, the numeric differences were small ${ }^{31} 3849$ and the significance disappeared at 3 months. ${ }^{31} 49$

Three studies including 149 patients reported SF-36 Physical Component Summary (SF-36 PCS) and Mental Component Summary (SF-36 MCS). Meta-analysis of SF-36 PCS and MCS did not detect significant differences at any time-point (from 6 weeks to 1 year, table 3 ).

Length of hospital stay and total cost

Only one $^{41}$ out of 10 studies $^{163032-343637414247}$ found a significant reduction in hospital length of stay (mean difference $=-0.8$-day). Meta-analysis of these studies did not detect significant differences in hospital length of stay for prehabilitation versus control (7 studies, 507 patients, WMD -0.3 days, $95 \%$ CI -0.8 to +0.1 days, figure 5 ).

Of the few studies ${ }^{37} 42$ that reported on costs, none reported significant reduction of overall costs with prehabilitation, but one ${ }^{42}$ described significantly increased physiotherapy costs with prehabilitation (mean difference=-£136.5). Even when total costs were converted to Canadian dollars and combined through meta-analysis, the results did not differ for prehabilitation versus none (2 studies, 242 patients, WMD $+\$ 0.5,95 \%$ CI $-\$ 384$ to $+\$ 393)$.

\section{Other outcomes}

Other outcomes of interest, including patient satisfaction, stroke, cardiovascular events and readmissions, were inadequately reported for meta-analysis. Adverse events and discontinuations were rarely reported within the studies; however, in at least one study, there was concern about increased cardiovascular events and stroke, and poorer SF-36 general health, energy and mental health among the withdrawn patients although the author stated no evidence that study withdrawal varied by group. ${ }^{42}$ In some studies, there were reports of patient withdrawals due to adverse events. ${ }^{34} 4249$ Some studies reported no significant postoperative complications between groups, ${ }^{35-37}$ no serious adverse events ${ }^{33} 3536$ or no adverse events. ${ }^{47}$

\section{Subgroup analysis and sensitivity analysis}

Effect sizes were similar between hip and knee replacement subgroups for WOMAC pain and function scores (see online supplementary table S3), as indicated by non-significant $p$ values for interaction.

Sensitivity analysis using SMD (instead of WMD), RoM and different thresholds for defining patient acceptable symptom state (20 and 40, instead of 30), and replacing function sub-score with total score, did not significantly change the results (see online supplementary tables S4 and S5).

While publication bias was not indicated for pain score, asymmetric funnel plots indicated the possibility of publication bias for function scores (see online supplementary figures S1 and figure S2, table 2).

\section{INTERPRETATION}

\section{Main findings}

Existing evidence from 22 randomised controlled trials suggests that prehabilitation for patients planning to 
Table 3 Summary of results for prehabilitation versus no prehabilitation

\begin{tabular}{|c|c|c|c|c|c|}
\hline Outcomes & Studies, (n) & Patients, (n) & Heterogeneity test $p$ value & $I^{2}(\%)$ & WMD and $95 \% \mathrm{CI}$ \\
\hline Pain at 4 weeks or less & 4 & 213 & 0.08 & 55 & $-6.1(-10.6 \text { to }-1.6)^{\star}$ \\
\hline Pain at $6-8$ weeks & 5 & 488 & 0.31 & 16 & $-1.4(-5.5$ to +2.6$)$ \\
\hline Pain at 12 weeks & 10 & 806 & 0.05 & 46 & $-2.9(-6.2$ to +0.3$)$ \\
\hline Pain at 24 weeks & 3 & 247 & 0.22 & 33 & $-2.5(-5.6$ to +0.6$)$ \\
\hline Pain at 1 year or more & 1 & 109 & NA & NA & $-2.0(-7.5$ to +3.5$)$ \\
\hline Function at 4 weeks or less & 5 & 257 & $<0.001$ & 79 & $-3.6(-7.7$ to +0.5$)$ \\
\hline Function at $6-8$ weeks & 5 & 488 & 0.21 & 31 & $-3.9(-7.6 \text { to }-0.3)^{*}$ \\
\hline Function at 12 weeks & 12 & 836 & $<0.001$ & 69 & $-4.0(-7.5 \text { to }-0.5)^{*}$ \\
\hline Function at 24 weeks & 5 & 345 & $<0.001$ & 89 & $-0.5(-5.8$ to +4.7$)$ \\
\hline Function at 1 year or more & 6 & 296 & 0.99 & 0 & $-0.6(-2.6$ to +145$)$ \\
\hline First days of climbing stairs (days) & 2 & 99 & 0.44 & 0 & $-1.4(-1.9 \text { to }-0.8)^{*}$ \\
\hline First days of walking (days) & 2 & 99 & 0.24 & 29 & $-0.2(-0.4$ to +0.002$)$ \\
\hline First days of use of toilet (days) & 2 & 99 & 0.87 & 0 & $-0.9(-1.3 \text { to }-0.5)^{*}$ \\
\hline First days of use of chair (days) & 2 & 99 & 0.50 & 0 & $-1.2(-1.7 \text { to }-0.8)^{*}$ \\
\hline SF-36 PCS at 6 weeks & 1 & 19 & NA & NA & $2.7(-9.4$ to +14.7$)$ \\
\hline SF-36 PCS at 12 weeks & 3 & 149 & 0.13 & 50 & $-0.3(-5.4$ to +4.7$)$ \\
\hline SF-36 PCS at 24 weeks & 1 & 109 & NA & NA & $0.0(-3.4$ to +3.4$)$ \\
\hline SF-36 PCS at 1 year & 1 & 109 & NA & NA & $-3.0(-6.4$ to +0.4$)$ \\
\hline SF-36 MCS at 6 weeks & 1 & 17 & NA & NA & $-3.4(-19.9$ to +13.0$)$ \\
\hline SF-36 MCS at 12 weeks & 3 & 149 & 0.72 & 0 & $-0.4(-3.7$ to +2.9$)$ \\
\hline SF-36 MCS at 24 weeks & 1 & 109 & NA & NA & $-1.0(-4.9$ to +2.9$)$ \\
\hline SF-36 MCS at 1 year & 1 & 109 & NA & NA & $-2.0(-5.1$ to +1.1$)$ \\
\hline Length of stay (days) & 7 & 507 & 0.68 & 0 & $-0.3(-0.8$ to +0.1$)$ \\
\hline Total cost (Canadian dollars) & 2 & 242 & 0.99 & 0 & $+5(-384$ to +393$)$ \\
\hline
\end{tabular}

Pain and function scores were converted to WOMAC (Western Ontario and McMaster Universities osteoarthritis index) 0-100 subscales, and high score indicates more pain or dysfunction.

${ }^{*} \mathrm{p}<0.05$.

MCS, mental component summary; NA, not applicable; PCS, physical component summary; WMD, weighted mean difference.

undergo joint replacement does not affect postoperative pain and function to a degree that would be considered clinically relevant; however, this is based on studies with significant limitations, providing very low certainty in estimates. While some differences reached statistical significance, the effects are too small to be considered clinically important (ie, an improvement of a few points on a scale of $0-100$ is likely clinically irrelevant, and undetectable to patients). Our analysis shows that prehabilitation reduced WOMAC pain score by 6 with $95 \%$ CI $(-10.6$ to -1.6$)$ within 4 weeks, and with no difference remaining beyond 4 weeks, which is generally smaller than the minimal clinically important improvement of at least 9.7 at 6 weeks, ${ }^{51}{ }^{52}$ even when the most optimistic extremes of the CIs are considered in our analysis. Even when the 'patient accepted pain state' was defined as achieving 30 in WOMAC pain subscale $0-100,{ }^{18-20}$ there was only an absolute increase of $3.9 \%$ of patients achieving this threshold. Similarly for function improvement, prehabilitation improved early function by $3.9-4.0$ points on the WOMAC function subscale $0-100$, which is much smaller than the threshold of minimally important difference, ranged from 7.9 to $25.9,{ }^{51-56}$ and only $1.3 \%$ to $5.4 \%$ more patients reached a WOMAC function score $\leq 30$. Although prehabilitation allowed patients to resume ADL 0.9-1.4 days earlier than no formal prehabilitation, the difference is trivial, and, importantly, very few studies reported on this time point (ie, 2 of 22 studies), which prevents definitive conclusions. Similarly, for the outcome of length of stay, there was no difference between groups, and if statistical significance had been achieved, the difference would have been only 0.3 days, which is a minimal difference. Jurisdictions considering implementation (or continuation) of prehabilitation services should consider whether resources could be better spent elsewhere on interventions of proven clinical benefit. Until sufficient evidence accrues to definitively conclude that prehabiliation provides meaningful benefit, investment in prehabilitation does not represent the best use of limited resources in a healthcare system where other opportunities with proven benefits could be funded instead.

\section{Relation to prior reviews}

Similar to this meta-analysis, most previous meta-analyse ${ }^{10}{ }^{11}$ and systematic reviews ${ }^{79} 12$ suggested that the impact of prehabilitation has not been proven by the existing evidence. In contrast to our analysis, Gill and McBurney ${ }^{8}$ suggested that exercise-based interventions reduce pain and improve physical function for people awaiting hip replacement surgery, but not those awaiting knee replacement surgery. It is notable that there were some limitations in the analysis by Gill and McBurney, ${ }^{8}$ wherein some included trials did not report 
Participants

ale $0-100$ Unclear conceal

indirectness imprecision (only 4

$(-10.6$

2.2\% patients

state of $\leq 30$

WOMAC pain scale

: Missing data $>15 \%:\left.3 \quad\right|^{2}=16 \%$

100; Iower values indicate less pain

No serious No serious Undetected

studies)

nclear concealment: 5 inconsistency;

due to risk of $\quad(-6.2$ to

achieved

pain scale

$C$ pain

(0-100) Unclear concealment: 2 inconsistency;

assessors not blinded: heterogeneity 0.22

WMD -2.5

due to risk of +0

MAC pain

$6.2 \%$ more

acceptable

on WOMAC

$(0-100)$

Continued 


\section{Summary of findings}

\section{Participants}

(studies)

Follow-up Risk of bias
488 (5 studies) Serious risk of bias* ${ }^{*}$ No serious No serious No serious Uncertain $\oplus \oplus \oplus \Theta$

6 to 8 weeks

$\begin{array}{ll}\text { Unclear concealment: } 3 & \text { inconsistency; } \\ \text { studies; outcome } & \text { p value on test for } \\ \text { assessors not blinded: } & \text { heterogeneity }=0.21\end{array}$

indirectness imprecision (only 5

studies)

2; missing data $>15 \%$ : $\left.3 \quad\right|^{2}=30 \%$

Relative

\section{effect or} CI) non-prehabilitation $(95 \% \mathrm{Cl})$

\section{$\oplus \oplus \oplus \Theta$}

WMD $-3.9 \quad 54.3 \%$ patients

$(-7.6$ to bias

achieved

state $\leq 30$ on acceptable function

WOMAC function scale $(0-100)$

\section{WMD $-4.0 \quad 62.6 \%$ patients}

$(-7.5$ to

$\begin{array}{lll}836(12 & \text { Serious risk of bias* } & \text { Serious } \\ \text { studies) } & \text { Unclear concealment: } 6 & \text { inconsistency; } \\ 12 \text { weeks } & \text { studies; outcome } & \text { p value on test for } \\ & \text { assessors not blinded: } & \text { heterogeneity } \\ & 4 ; \text { missing data }>15 \%: 6 & <0.001, \mathrm{I}^{2}=69 \%\end{array}$

cale 0-100; lower values indicate better function

asymmetry

on funnel

VERY LOW

plot; Egger's bias,

test $\mathrm{p}=0.04$ inconsistency

and

publication

bias

Function score at 24 weeks, measured with: WOMAC function subscale 0-100; Lower values indicate better function

$\begin{array}{lll}345 \text { (7 studies) } & \text { Serious risk of bias }{ }^{*} & \text { Serious } \\ 24 \text { weeks } & \text { Unclear concealment: } 4 & \text { inconsistency; } \\ & \text { studies; outcome } & \text { p value on test for }\end{array}$

assessors not blinded: heterogeneity

2; missing data $>15 \%$ : $2<0.001, \mathrm{I}^{2}=89 \%$
No serious No serious Uncertain

indirectness imprecisiont (only 7

studies)

\section{$\oplus \oplus \Theta \Theta$ LOW WMD $-\mathbf{0 . 5}$}

due to risk of $\quad(-5.8$ to

bias and

inconsistency

*None of the studies in the meta-analyses blinded patients and only 2 studies blinded the care providers.

†We did not rate down due to imprecision although $95 \% \mathrm{Cl}$ includes no effect because either extreme of the $95 \% \mathrm{Cl}$ is too small to be clinically important difference.

WMD, weighted mean differences; WOMAC, Western Ontario and McMaster Universities Osteoarthritis Index. 


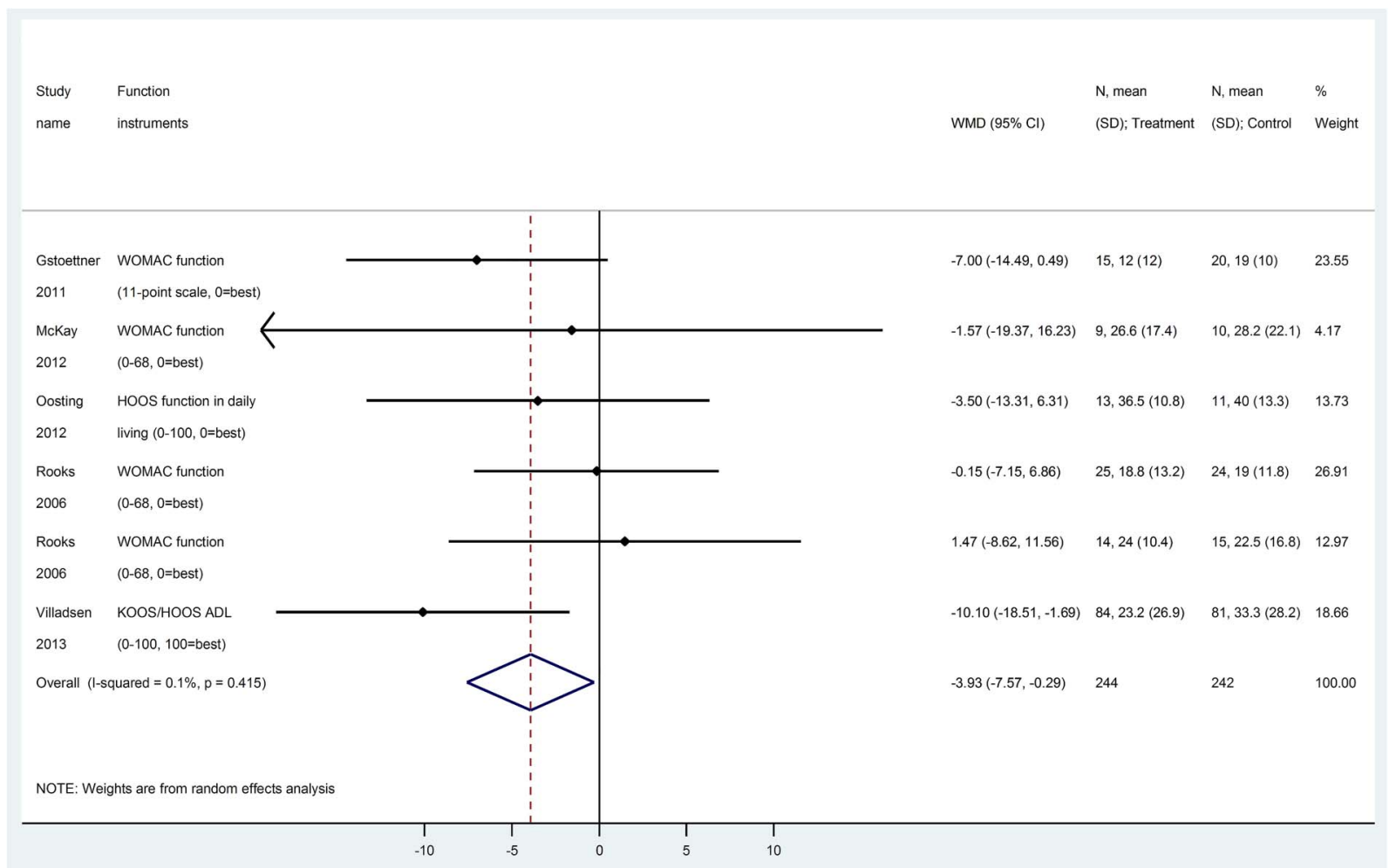

Figure 3 Function score at 6-8 weeks (converted to WOMAC function subscale 0-100) for prehabilitation versus no prehabilitation in joint replacement surgery. WOMAC, Western Ontario and McMaster Universities Osteoarthritis Index.

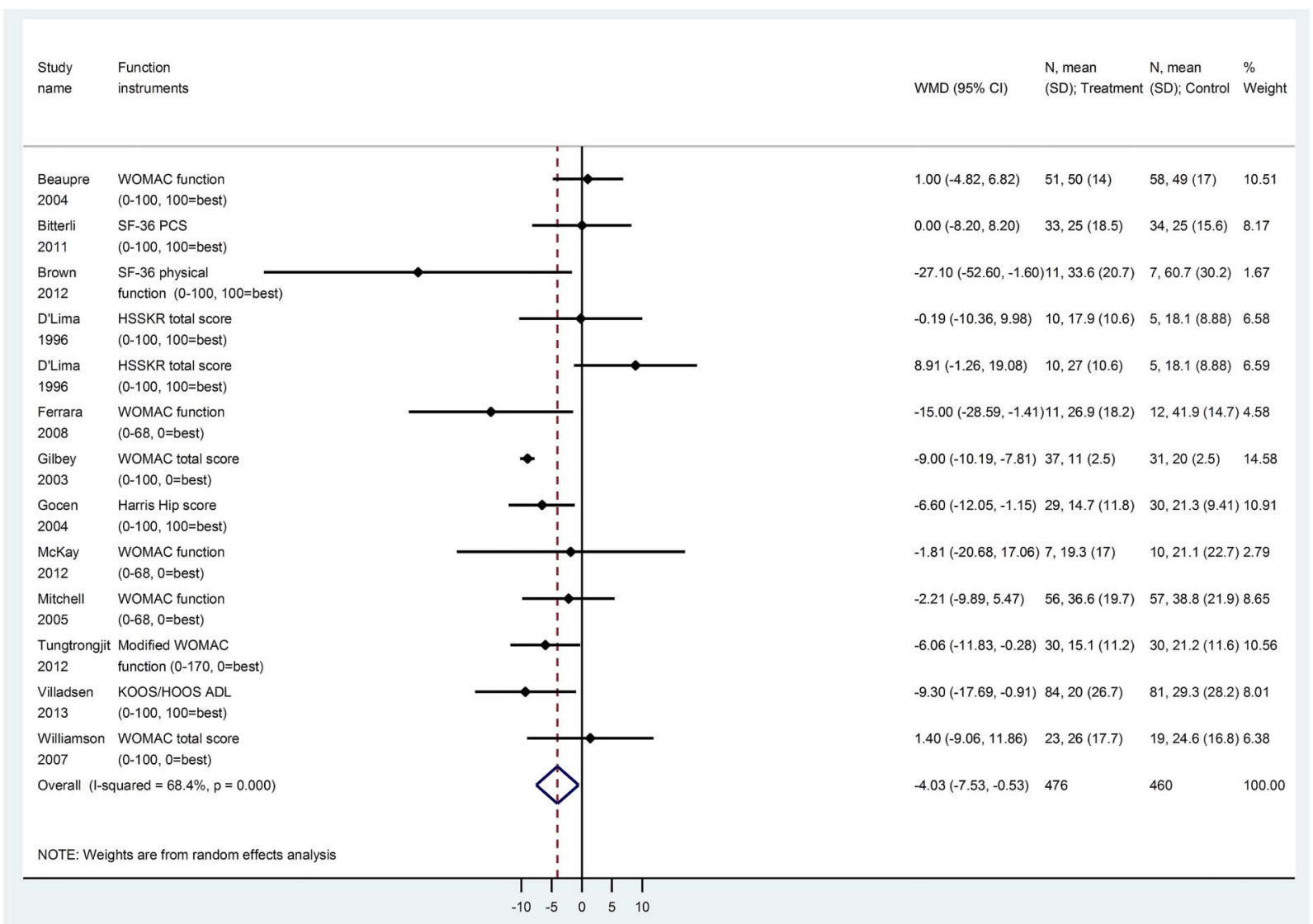

Figure 4 Function score at 12 weeks (converted to WOMAC function subscale 0-100) for prehabilitation versus no prehabilitation in joint replacement surgery. ADL, activities of daily living; WOMAC, Western Ontario and McMaster Universities Osteoarthritis Index. KOOS, Knee injury and Osteoarthritis Outcome Score; HOOS, Hip disability and Osteoarthritis Outcome Score. 
Study

name
$\mathrm{N}$, mean $\quad \mathrm{N}$, mean $\quad \%$

WMD $(95 \% \mathrm{Cl}) \quad$ (SD); Treatment (SD); Control Weight

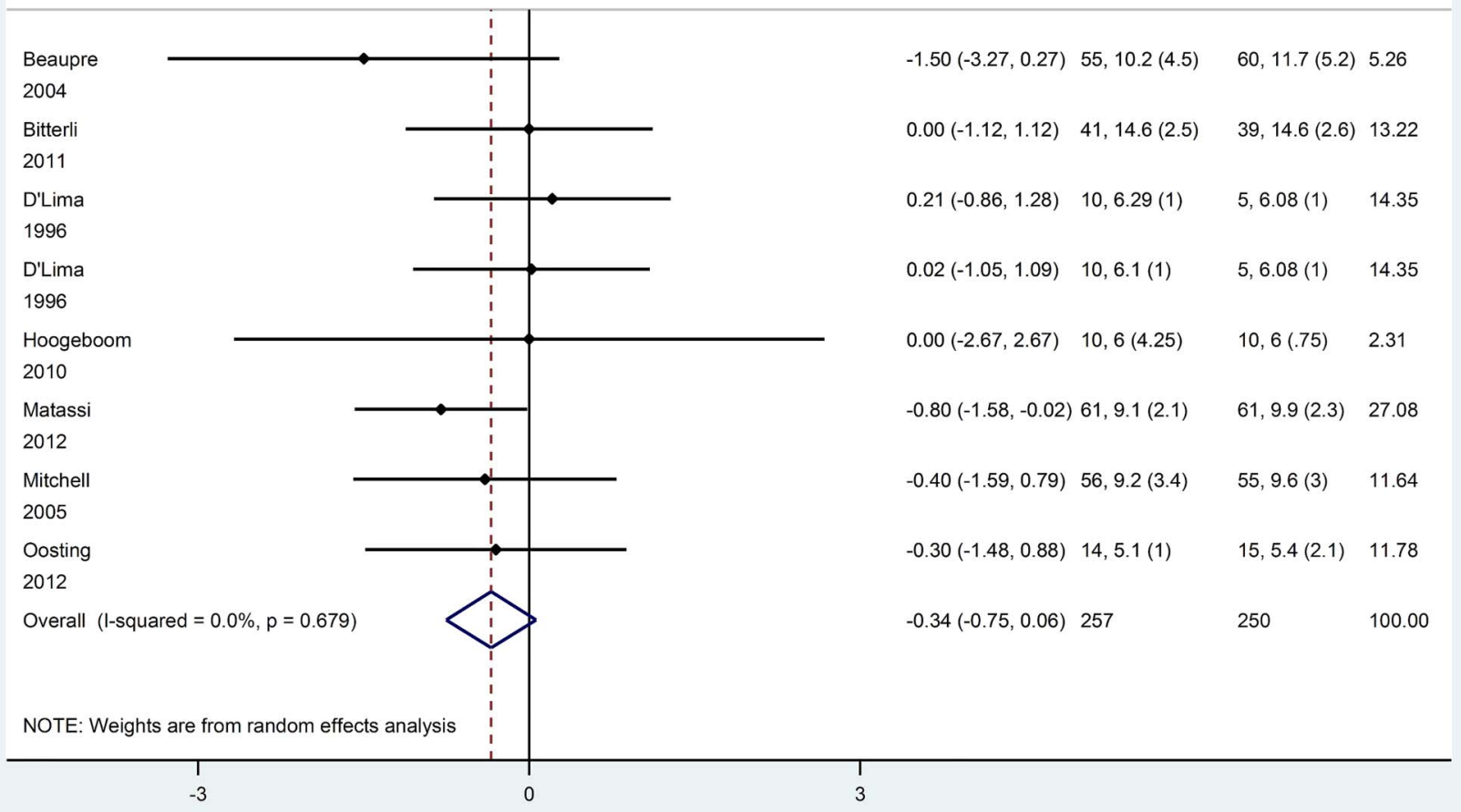

Figure 5 Hospital length of stay (days) for prehabilitation versus no prehabilitation in joint replacement surgery.

if the patients underwent surgery after the intervention, ${ }^{57} 58$ and/or failed to report postoperative outcomes, ${ }^{59} 60$ and one included trial allocated patients based on the geographic availability, which may have introduced selection bias and unit of measurement errors. Furthermore, a total of nine relevant trials $^{30343840-42444550}$ were not included in Gill (2013).

\section{Strengths and limitations}

Strengths of this review include rigorous methodology, including the comprehensive systematic search without limits by language, date or publication status, which identified seven randomised controlled trials $^{30} 343840414550$ not included in any previous systematic reviews. ${ }^{6-13}$ Furthermore, we analysed the effect of prehabilitation by converting to a standardised measurement of WOMAC pain and function scores, and used different presentation methods to enhance interpretability and to improve ability to find potential signals in effect size through meta-analysis, ${ }^{61}$ which is beyond what other systematic reviews published. In addition, this meta-analysis addressed all available clinically relevant outcomes, while previous reviews addressed only a few selected outcomes. Application of GRADE for rating quality of evidence provides improved context for interpreting the findings in light of inherent strengths and limitations of the included studies. ${ }^{62} 63$
There were a number of specific limitations in the existing clinical trials comparing prehabilitation with control. The most significant limitation is the lack of large randomised controlled trials conducted in this area. Included studies were small (median 81 patient, ranging from 21 to 165 ), of relatively short duration of follow-up (median 3 months, ranging from 4 weeks to 1 year), and many of them provided inadequate description of the frequency, intensity and duration of prehabilitation provided. Definitions for prehabilitation and for outcomes measurements were heterogeneous across studies. Patient compliance with prehabilitation was reported as $75 \%$ in three studies, ${ }^{41}{ }^{42} 50$ and $90 \%$ or greater in seven studies, ${ }^{17} 303335364348$ and was not reported in the remainder of the studies. Most studies provided an inadequate description of the components of the prehabilitation programmes provided, and few described the fidelity of programme implementation. ${ }^{64}$ Future studies in this area should follow current guidelines for intervention description (TIDieR checklist) to enable transparent evaluation and replication of programmes. ${ }^{65}$ In a number of studies, cointerventions were provided in the prehabilitation (eg, education) and, in some cases, these cointerventions were not provided in the control group. ${ }^{31} 32{ }^{34}$ Nevertheless, this would likely provide an overestimate of the potential benefit for prehabilitation; despite this potential positive bias, still no differences were found for prehabilitation. Considered 
together, the heterogeneity of the included studies in types of prehabilitation programmes, control group interventions, compliance and fidelity within the programmes, and systematic differences in the study population, likely impacted the ability to detect differences, if any exist. Although we performed subgroup analysis for hip versus knee replacement surgery, this failed to explain the heterogeneity across studies. Owing to the limited numbers of studies, meta-analysis was not performed for the effect of different types of prehabilitation (eg, exercise only vs exercise plus education). Publication bias was not detected; however, the methodological quality of included studies is very low, which was the major reason that we downgraded the overall quality of evidence. The high risk of bias, combined with the selective reporting of important outcomes across the studies (eg, only two studies reported time to return to ADL and total costs) precludes definitive conclusions, despite at least 22 randomised controlled trials being conducted.

\section{CONCLUSION}

Existing evidence suggests that, in patients undergoing joint replacement, the effect of prehabilitation (exercise/physiotherapy programmes in the months prior to surgery) on pain and function are too small to be considered clinically-important and were not robust over time. Prehabilitation did not result in clinically important (or statistically significant) differences in most measures of patient recovery, quality of life, length of stay and costs. Future research of sufficient power to measure clinically-relevant outcomes is required to identify which, if any, form of prehabilitation achieves better outcomes than in these trials. Jurisdictions considering implementation of prehabilitation services should consider whether resources could be better spent elsewhere on interventions of proven clinical benefit.

\author{
Author affiliations \\ ${ }^{1}$ Centre for Medical Evidence, Decision Integrity and Clinical Impact (MEDICI), \\ University of Western Ontario, London, Ontario, Canada \\ ${ }^{2}$ Chinese Cochrane Centre, West China Hospital, Sichuan University, Chengdu, \\ China \\ ${ }^{3}$ Michael G DeGroote Institute for Pain Research and Care, McMaster \\ University, Hamilton, Ontario, Canada \\ ${ }^{4}$ Department of Anesthesiology and Pain Medicine, Konkuk University School \\ of Medicine, Chungju, South Korea \\ ${ }^{5}$ Department of Anesthesiology, Fuwai Hospital, Chinese Academy of Medical \\ Sciences and Peking Union Medical College, Beijing, China \\ ${ }^{6}$ Department of Anesthesia \& Perioperative Medicine, University of Western \\ Ontario, London, Ontario, Canada \\ ${ }^{7}$ Department of Epidemiology \& Biostatistics, University of Western Ontario, \\ London, Ontario, Canada
}

Acknowledgements The authors would like to thank Gordon Guyatt, MD, for mentorship to Li Wang on methodology, and Amy Newitt, MLIS, for her contributions to the search updates and manuscript preparation. The authors are also thankful to 'National Natural Science Foundation of China' (Project \# 71073105) for supporting LW in the methodology training.

Contributors LW contributed to the study conception and design, literature screening, acquisition of data, analysis and interpretation of data, drafting of the manuscript and revision based on the comments of the co-authors. ML and ZZ participated in the literature screening, data acquisition and critical revision of the manuscript. JM performed the literature search, article retrieval and data acquisition. DC contributed to study conception and design, and critical revision of the manuscript. JM guided the methodology, and contributed to the study conception and design, data checking and interpretation, drafting and critical revision of the manuscript. All the authors approved the version submitted for publication and agreed to act as guarantors of the work.

Funding This study was supported by the MEDICI Centre, Department of Anesthesia and Perioperative Medicine, London Health Sciences Center, St Joseph's Healthcare London, Lawson Health Research Institute, and the Schulich School of Medicine and Dentistry, University of Western Ontario. In addition, funding was provided in part by 'AMOSO Innovation Fund' (Project \#INN 11-008, to JM and DC).

Competing interests None declared.

Provenance and peer review Not commissioned; externally peer reviewed.

Data sharing statement No additional data are available.

Open Access This is an Open Access article distributed in accordance with the Creative Commons Attribution Non Commercial (CC BY-NC 4.0) license, which permits others to distribute, remix, adapt, build upon this work noncommercially, and license their derivative works on different terms, provided the original work is properly cited and the use is non-commercial. See: http:// creativecommons.org/licenses/by-nc/4.0/

\section{REFERENCES}

1. Felson DT, Lawrence RC, Hochberg MC, et al. Osteoarthritis: New insights. part 2: treatment approaches. Ann Intern Med 2000;133:726-37.

2. Beswick AD, Wylde V, Gooberman-Hill R, et al. What proportion of patients report long-term pain after total hip or knee replacement for osteoarthritis? A systematic review of prospective studies in unselected patients. BMJ Open 2012;2:e000435.

3. Vissers MM, Bussmann JB, Verhaar JA, et al. Recovery of physical functioning after total hip arthroplasty: Systematic review and meta-analysis of the literature. Phys Ther 2011;91:615-29.

4. Nilsdotter AK, Toksvig-Larsen S, Roos EM. Knee arthroplasty: Are patients' expectations fulfilled? A prospective study of pain and function in 102 patients with 5-year follow-up. Acta Orthop 2009;80:55-61.

5. Mak JC, Fransen M, Jennings M, et al. Evidence-based review for patients undergoing elective hip and knee replacement. ANZ J Surg 2014;84:17-24.

6. Ackerman IN, Bennell KL. Does pre-operative physiotherapy improve outcomes from lower limb joint replacement surgery? A systematic review. Aust J Physiother 2004;50:25-30.

7. Jordan RW, Smith NA, Chahal GS, et al. Enhanced education and physiotherapy before knee replacement; is it worth it? A systematic review. Physiotherapy 2014;100:305-12.

8. Gill SD, McBurney H. Does exercise reduce pain and improve physical function before hip or knee replacement surgery? A systematic review and meta-analysis of randomized controlled trials. Arch Phys Med Rehabil 2013;94:164-76.

9. Shoemaker MJ, Gibson C, Saagman S. Preoperative exercise in individuals undergoing total knee arthroplasty: state of the evidence. Top Geriatr Rehabil 2013;29:2-16.

10. Hoogeboom TJ, Oosting E, Vriezekolk JE, et al. Therapeutic validity and effectiveness of preoperative exercise on functional recovery after joint replacement: A systematic review and meta-analysis. PLOS ONE 2012;7:e38031.

11. Wallis JA, Taylor NF. Pre-operative interventions (non-surgical and non-pharmacological) for patients with hip or knee osteoarthritis awaiting joint replacement surgery-a systematic review and meta-analysis. Osteoarthritis Cartilage 2011;19:1381-95.

12. Barbay K. Research evidence for the use of preoperative exercise in patients preparing for total hip or total knee arthroplasty. Orthop Nurs 2009;28:127-33.

13. Lucas B. Does a pre-operative exercise programme improve mobility and function post-total knee replacement: a mini-review. J Orthop Nurs 2004;8:25-33.

14. Higgins JPT, Green S ed. Cochrane handbook for systematic reviews of interventions Version 5.1.0 [updated March 2011]. The Cochrane Collaboration, 2011. http://www.cochrane-handbook.org 
15. Thorlund K, Walter SD, Johnston BC, et al. Pooling health-related quality of life outcomes in meta-analysis-a tutorial and review of methods for enhancing interpretability. Res Synth Methods 2011;2:188-203.

16. D'Lima DD, Colwell CW Jr, Morris BA, et al. The effect of preoperative exercise on total knee replacement outcomes. Clin Orthop Relat Res 1996;326:174-82.

17. Gilbey HJ, Ackland TR, Wang AW, et al. Exercise improves early functional recovery after total hip arthroplasty. Clin Orthop Relat Res 2003;408:193-200.

18. Tubach F, Ravaud P, Baron G, et al. Evaluation of clinically relevant states in patient reported outcomes in knee and hip osteoarthritis: The patient acceptable symptom state. Ann Rheum Dis 2005;64:34-7.

19. Tubach F, Ravaud P, Martin-Mola E, et al. Minimum clinically important improvement and patient acceptable symptom state in pain and function in rheumatoid arthritis, ankylosing spondylitis, chronic back pain, hand osteoarthritis, and hip and knee osteoarthritis: Results from a prospective multinational study. Arthritis Care Res (Hoboken) 2012;64:1699-707.

20. Escobar A, Gonzalez M, Quintana JM, et al. Patient acceptable symptom state and OMERACT-OARSI set of responder criteria in joint replacement. identification of cut-off values. Osteoarthritis Cartilage 2012;20:87-92

21. Maxwell JL, Felson DT, Niu J, et al. Does clinically important change in function after knee replacement guarantee good absolute function? the multicenter osteoarthritis study. J Rheumatol 2014;41:60-4.

22. Anzures-Cabrera J, Sarpatwari A, Higgins JP. Expressing findings from meta-analyses of continuous outcomes in terms of risks. Stat Med 2011;30:2967-85.

23. Guyatt GH, Oxman AD, Santesso N, et al. GRADE guidelines: 12. preparing summary of findings tables-binary outcomes. J Clin Epidemiol 2013;66:158-72.

24. Guyatt G, Oxman AD, Akl EA, et al. GRADE guidelines: 1. introduction-GRADE evidence profiles and summary of findings tables. J Clin Epidemiol 2011;64:383-94.

25. Guyatt GH, Oxman AD, Montori V, et al. GRADE guidelines: 5. rating the quality of evidence-publication bias. J Clin Epidemiol 2011;64:1277-82.

26. Guyatt GH, Oxman AD, Kunz R, et al. GRADE guidelines 6. rating the quality of evidence-imprecision. J Clin Epidemiol 2011;64:1283-93.

27. Guyatt GH, Oxman AD, Kunz R, et al. GRADE guidelines: 7. rating the quality of evidence-inconsistency. J Clin Epidemiol 2011;64:1294-302.

28. Guyatt $\mathrm{GH}$, Oxman AD, Kunz R, et al. GRADE guidelines: 8. rating the quality of evidence-indirectness. J Clin Epidemiol 2011;64:1303-10.

29. Guyatt GH, Thorlund K, Oxman AD, et al. GRADE guidelines: 13 preparing summary of findings tables and evidence profiles-continuous outcomes. J Clin Epidemiol 2013;66:173-83.

30. Bitterli $\mathrm{R}$, Sieben JM, Hartmann $\mathrm{M}$, et al. Pre-surgical sensorimotor training for patients undergoing total hip replacement: a randomised controlled trial. Int J Sports Med 2011;32:725-32.

31. Ferrara PE, Rabini A, Maggi L, et al. Effect of pre-operative physiotherapy in patients with end-stage osteoarthritis undergoing hip arthroplasty. Clin Rehabil 2008;22:977-86.

32. Gocen Z, Sen A, Unver B, et al. The effect of preoperative physiotherapy and education on the outcome of total hip replacement: a prospective randomized controlled trial. Clin Rehabil 2004;18:353-8.

33. Hoogeboom TJ, Dronkers JJ, van den Ende $\mathrm{CH}$, et al. Preoperative therapeutic exercise in frail elderly scheduled for total hip replacement: a randomized pilot trial. Clin Rehabil 2010;24 901-10.

34. Vukomanović A, Popović Z, Durović A, et al. The effects of short-term preoperative physical therapy and education on early functional recovery of patients younger than 70 undergoing total hip arthroplasty. Vojnosanit Pregl 2008;65:291-7.

35. Wang AW, Gilbey HJ, Ackland TR. Perioperative exercise programs improve early return of ambulatory function after total hip arthroplasty: a randomized, controlled trial. Am J Phys Med Rehabil 2002;81:801-6.

36. Oosting E, Jans MP, Dronkers JJ, et al. Preoperative home-based physical therapy versus usual care to improve functional health of frail older adultsscheduled for elective total hip arthroplasty: a pilot randomized controlled trial. Arch Phys Med Rehabil 2012;93:610-16

37. Beaupre L, Lier D, Davies D, et al. The effect of a preoperative exercise and education program on functional recovery, health related quality of life, and health service utilization following primary total knee arthroplasty. J Rheumatol 2004;31:1166-73.

38. Brown K, Top R, Brosky JA, et al. Prehabilitation and quality of life three months after total knee arthroplasty: a pilot study. Percept Mot Skills 2012;115:765-74.

39. Evgeniadis G, Beneka A, Malliou P, et al. Effects of pre- or postoperative therapeutic exercise on the quality of life, before and after total knee arthroplasty for osteoarthritis. J Back Musculoskelet Rehabil 2008;21:161-9.

40. Gstoettner M, Raschner C, Dirnberger E, et al. Preoperative proprioceptive training in patients with total knee arthroplasty. Knee 2011;18:265-70.

41. Matassi F, Duerinckx J, Vandenneucker $\mathrm{H}$, et al. Range of motion after total knee arthroplasty: the effect of a preoperative home exercise program. Knee Surg Sports Traumatol Arthrosc 2014;22:703-9.

42. Mitchell C, Walker J, Walters S, et al. Costs and effectiveness of pre- and post-operative home physiotherapy for total knee replacement: randomizedcontrolled trial. J Eval Clin Pract 2005;11:283-92.

43. McKay $\mathrm{C}$, Prapavessis $\mathrm{H}$, Doherty $\mathrm{T}$. The effect of a prehabilitation exercise program on quadriceps strength for patients undergoing total knee arthroplasty: a randomized controlled pilot study. PM $R$ 2012;4:647-56.

44. Topp R, Swank AM, Quesada PM, et al. The effect of prehabilitation exercise on strength and functioning after total knee arthroplasty. PM $R$ 2009;1:729-35.

45. Tungtrongjit $Y$, Weing $P$, Saunkool $P$. The effect of preoperative quadriceps exercise on functional outcomes after total knee arthroplasty. J Med Assoc Thai 2012;95(Suppl 10):S58-66.

46. Weidenhielm L, Mattsson E, Brostrom L, et al. Effect of preoperative physiotherapy in uncompartmental prosthetic knee replacement. Scand J Rehab Med 1993;25:33-9.

47. Williamson L, Wyatt MR, Yein $\mathrm{K}$, et al. Severe knee osteoarthritis: a randomized controlled trial of acupuncture, physiotherapy (supervised exercise) and standard management for patients awaiting knee replacement. Rheumatology (Oxford) 2007;46:1445-9.

48. Rooks DS, Huang J, Bierbaum BE, et al. Effect of preoperative exercise on measures of functional status in men and women undergoing total hip and knee arthroplasty. Arthritis Rheum. 2006;55:700-8

49. Villadsen A, Overgaard S, Holsgaard-Larsen A, et al. Postoperative effects of neuromuscular exercise prior to hip or knee arthroplasty: a randomised controlled trial. Ann Rheum Dis. 2014;73 1130-7.

50. Villadsen A, Overgaard S, Holsgaard-Larsen A, et al. Immediate efficacy of neuromuscular exercise in patients with severe osteoarthritis of the hip or knee: a secondary analysis from a randomized controlled trial. J Rheumatol 2014;41:1385-94.

51. Ehrich EW, Davies GM, Watson DJ, et al. Minimal perceptible clinical improvement with the western ontario and McMaster universities osteoarthritis index questionnaire and global assessments in patients with osteoarthritis. J Rheumatol 2000;27:2635-41.

52. Escobar A, Quintana JM, Bilbao A, et al. Responsiveness and clinically important differences for the WOMAC and SF-36 after total knee replacement. Osteoarthritis Cartilage 2007;15:273-80.

53. Angst F, Aeschlimann A, Michel BA, et al. Minimal clinically important rehabilitation effects in patients with osteoarthritis of the lower extremities. J Rheumatol 2002;29:131-8.

54. Escobar A, Garcia Pérez L, Herrera-Espiñeira C, et al. Total knee replacement; minimal clinically important differences and responders. Osteoarthritis Cartilage 2013;21:2006-12.

55. Quintana JM, Escobar A, Bilbao A, et al. Responsiveness and clinically important differences for the WOMAC and SF-36 after hip joint replacement. Osteoarthritis Cartilage 2005;13:1076-83.

56. Tubach F, Ravaud P, Baron G, et al. Evaluation of clinically relevant changes in patient reported outcomes in knee and hip osteoarthritis: the minimal clinically important improvement. Ann Rheum Dis 2005;64:29-33.

57. Börjesson M, Robertson E, Weidenheilm L, et al. Physiotherapy in knee osteoarthrosis: effect on pain and walking. Physiother Res Int 1996;1:89-97.

58. Nuñez M, Nuñez E, Segur JM. The effect of an educational program to improve health-related quality of life in patients with osteoarthritis on waiting list for total knee replacement: a randomized study. Osteoarthritis Cartilage 2006;14:279-85

59. Aoki O, Tsumura N, Kimura A, et al. Home stretching exercise is effective for improving knee range of motion and gait in patients with knee osteoarthritis. J Phys Ther Sci 2009;21:113-19. 
60. Swank AM, Kachelman JB, Bibeau W. Prehabilitation before total knee arthroplasty increases strength and function in older adults with severe osteoarthritis. J Strength Cond Res 2011;25:318.

61. Johnston BC, Bandayrel K, Friedrich JO, et al. Presentation of continuous outcomes in meta-analysis: a survey of clinicians' understanding and preferences. Quebec City, Canada: 21st Cochrane Colloquium, 2013.

62. Guyatt GH, Oxman AD, Vist GE, et al. GRADE: An emerging consensus on rating quality of evidence and strength of recommendations. BMJ 2008;336:924-6.
63. Langer G, Meerpohl JJ, Perleth M, et al. [GRADE guidelines:

1. introduction-GRADE evidence profiles and summary of findings tables]. Z Evid Fortbild Qual Gesundhwes 2012;106:357-68

64. Taylor KL, Weston M, Batterham AM. Evaluating intervention fidelity: an example from a high-intensity interval training study. PLOS ONE 2015;10:e0125166.

65. Hoffmann TC, Glasziou PP, Boutron I, et al. Better reporting of interventions: template for intervention description and replication (TIDieR) checklist and guide. BMJ 2014;348:g1687. 